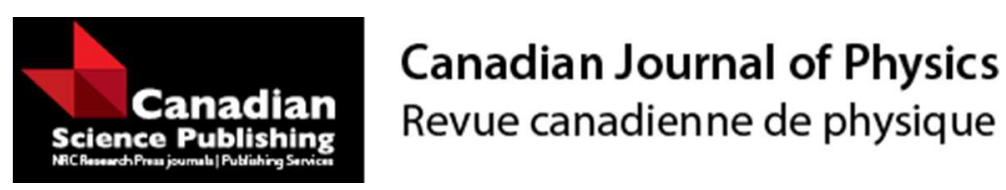

\title{
On mode matching analysis of fluid-structure coupled wave scattering between two flexible waveguides
}

\begin{tabular}{|r|l|}
\hline Journal: & Canadian Journal of Physics \\
\hline Manuscript ID & cjp-2016-0801.R1 \\
\hline Manuscript Type: & Article \\
\hline Date Submitted by the Author: & 03-Feb-2017 \\
\hline Complete List of Authors: & $\begin{array}{l}\text { Afzal, Muhammad; Capital University of Science and Technology, } \\
\text { Mathematics } \\
\text { Shafique, Sajid; Capital University of Science and Technology, Mathematics } \\
\text { Nawaz, Rab; COMSATS Institute of Information Technology, Mathematics }\end{array}$ \\
\hline Keyword: & $\begin{array}{l}\text { Scattering, Flexible waveguide, Non-Sturm-Liouville, Mode-Matching., } \\
\text { Energy flux }\end{array}$ \\
\hline &
\end{tabular}




\title{
On mode matching analysis of fluid-structure coupled wave scattering between two flexible waveguides
}

\author{
${ }^{a}$ Sajid Shafique, ${ }^{a}$ Muhammad Afzal, ${ }^{b}$ Rab Nawaz ${ }^{1}$ \\ ${ }^{a}$ Department of Mathematics, Capital University of Science and Technology, Islamabad, \\ Pakistan.

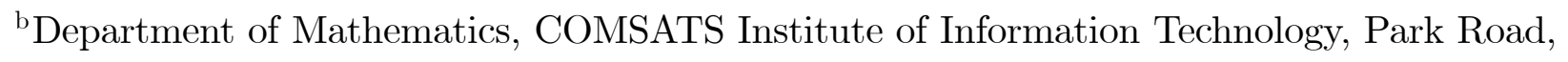 \\ Islamabad, Pakistan.
}

\begin{abstract}
A mode-matching analysis articulates the coupled wave scattering in a $2 \mathrm{D}$ waveguide structure. An elastic plate is attached at the upper surface parallel to the axis of inlet/outlet ducts whereas a flanged junction is introduced between two flexible waveguides. The main intention is to see that how choice of appropriate edge conditions and the incident forcing affect the scattered field for both structure-borne and fluid-borne vibrations. The graphical results illustrate to draw different physical conclusions. Through apposite numerical results, we observe the power distributions in attenuated regions with abrupt changes of height. Such configurations are directly relevant to the models of fluid/structural interactions.
\end{abstract}

Keywords: Scattering, flexible waveguide, non-Sturm-Liouville, mode-matching.

Subject classification: 02.30.Jr, 03.65.Nk, 42.25.Bs, 42.25.Fx, 43.20.Bi.

\section{Introduction}

The study of non-uniform obstacles in uniform waveguide structures has received wide attention in the literature. The transmission of elastic and electromagnetic waves, underwater sound propagation, and sound scattering in ducts or pipes are the major applications to such studies. The curiosity is to reduce the ducted fan noise emanated from aero engines, power stations and heating, ventilation, and air conditioning (HVAC) systems. Numerous investigations have been made to explore reduction of unwanted noise from different types of obstacles [1-6]. Several mathematical models and techniques exist for computing sound attenuation from different sources; see for example [7-10]. Cummings and Chang [11] imposed continuity of pressure and velocity over the inlet and outlet planes of the silencer while using

\footnotetext{
${ }^{1}$ Corresponding author e-mail address: rabnawaz@comsats.edu.pk, Ph: +92 (51) 90495534.
}

https://mc06.manuscriptcentral.com/cjp-pubs 
the eigenmodes in an analytic mode matching scheme. Peat [12], and later by Kirby [13], proposed more efficient alternative to Cummings and Chang's method. They established closed form analytic solutions based on the attenuation of the fundamental mode only. The methods proposed by Peat and Kirby are, however, precise only over a limited frequency range for a given waveguide structure. Such restrictions apply also to other methods based on the fundamental mode, for example methods proposed by Panigrahi and Munjal [14]. Recently Afzal et al [10] and, Nawaz and Lawrie [15], included the second mode forcing term which aim to carry energy through fluid-borne instead of structure-borne mode. It is thus established that the numerical results contrast well for both, the fundamental mode and secondary mode incidents.

Albeit, the main purpose of this article is to examine the wave scattering of two dimensional waveguide problems with wave bearing boundaries. Two flexible duct regions are connected vertically with a rigid flanged junction and a soft back strip. Such type of configurations are used in modelling of different modified silencers. In recent times, number of modelling techniques are studied for any shape or size of waveguide structure. These techniques involved both analytical as well as numerical approaches depending upon the complexity of model. Finite element method [16-17], boundary element method [18], iterative Newton Raphson scheme, Wiener-Hopf technique [19] and some analytic closed form solutions [20] are developed to deal with such configurations with their relative merits. The problem under consideration is solved using mode-matching approach subject to different set of edge conditions. The article is organized as follows:

The boundary value problem is stated and formulated in section 2. The resulting algebraic systems formulated in terms of reflected and transmitted coefficients are obtained while using the well-known semi analytic mode-matching approach. The incident forcing (corresponds to both structure and fluid borne modes) is considered so that its amplitude is unity. The solution to the underlying problem is presented in section 3 followed by subsections examining the dispersion and orthogonality relations, mode-matching and the use of three types of edge conditions. The distribution of reflected and transmitted powers is examined by testing few numerical illustrations in section 4 whereas results are concluded in section (5). 


\section{Mathematical formulation in 2D Duct}

Consider a two-dimensional infinite waveguide containing two semi-infinite duct regions $-\infty<\bar{x} \leq \overline{0}, \overline{0} \leq \bar{y} \leq \bar{a}$ and $\overline{0} \leq \bar{x}<\infty, \quad \bar{h}_{1} \leq \bar{y} \leq \bar{b}$, where overbar shows the dimensional setting of Cartesian coordinates. These duct regions are bounded below by an acoustically rigid surface at $\bar{y}=\overline{0}, \bar{h}_{1}$ whilst bounded above by an elastic plate at $\bar{y}=\bar{a}$, $-\infty<\bar{x} \leq \overline{0}$ and $\bar{y}=\bar{b}, \overline{0} \leq \bar{x}<\infty$. Two duct regions are mutually joined by means of two vertical strips lying at $\bar{x}=\overline{0}, \overline{0} \leq \bar{y} \leq \bar{h}_{2}$ and $\bar{x}=\overline{0}, \bar{a} \leq \bar{y} \leq \bar{b}$. The material properties on the sides of the vertical strips are assumed to be different. The sides of vertical strips lying at $\bar{x}=\overline{0}^{-}$are acoustically rigid while the sides aliened along $\bar{x}=\overline{0}^{+}$are soft. The interior region of the waveguide is filled with a compressible fluid of density $\rho$ and sound speed $c$, whereas, the exterior region is assumed to be in vaccu. The waveguide structure of the problem is shown in Figure (1).

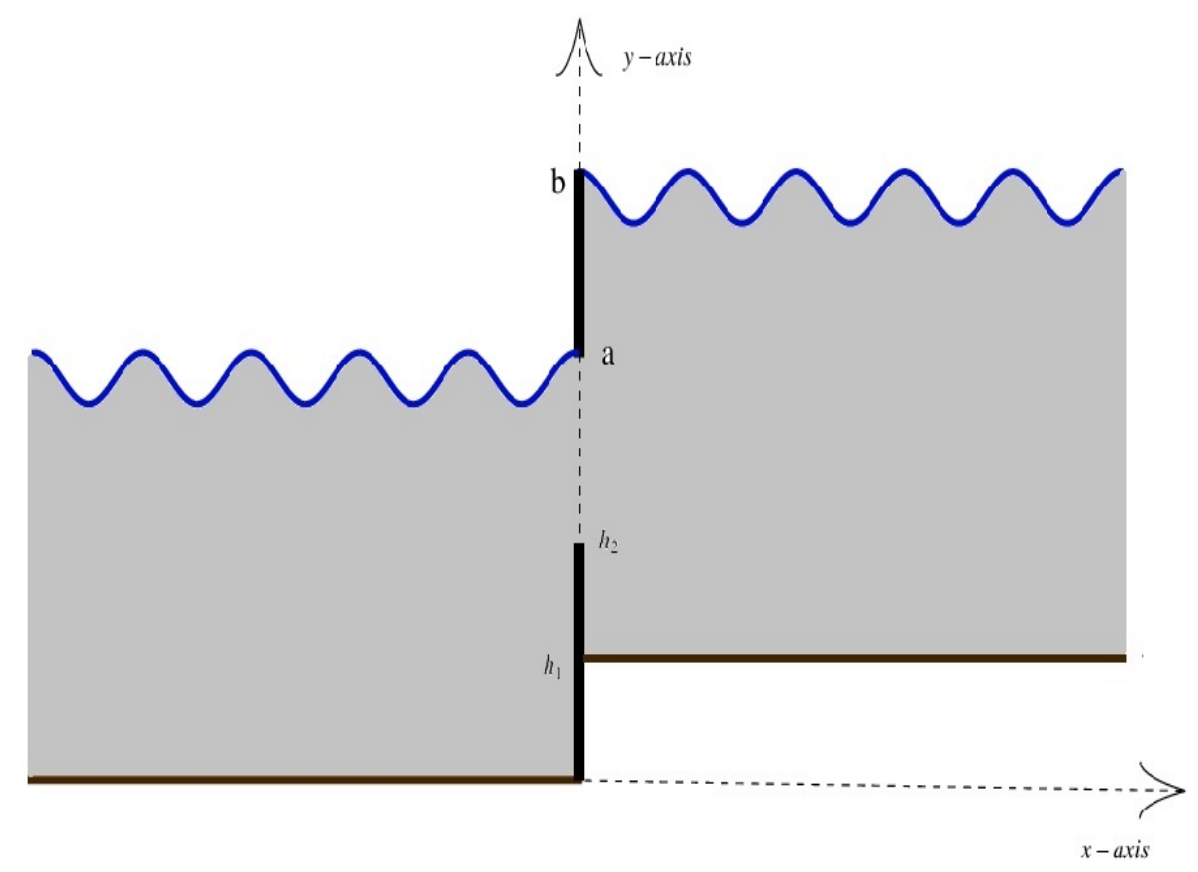

Figure (1): The physical configuration of the waveguide structure.

Assuming the harmonic time dependence as $e^{-i \omega \bar{t}}$, where $\omega=c k$ is the angular velocity and $k=2 \pi / f$ is the wave number. On non-dimensionalizing the boundary value problem with respect to the length scale $k^{-1}$ and the time scale $\omega^{-1}$ under the transformations $x=k \bar{x}$, 
$y=k \bar{y}$ and $t=\omega \bar{t}$, the Helmholtz's equation is governed as

$$
\left(\frac{\partial^{2}}{\partial x^{2}}+\frac{\partial^{2}}{\partial y^{2}}+1\right) \psi(x, y)=0
$$

For the convenience the time independent velocity potential $\psi$ (dimensionless) for two duct region is expressed in terms of two different scattered fields as

$$
\psi(x, y)=\left\{\begin{array}{ll}
\psi_{1}(x, y), & 0 \leq y \leq a, x \leq 0 \\
\psi_{2}(x, y), & h_{1} \leq y \leq b, x \geq 0
\end{array} .\right.
$$

At $y=0, h_{1}$, the rigid horizontal lower boundaries can be defined as

$$
\frac{\partial \psi_{j}}{\partial y}=0, \quad x \in \mathbb{R}, \text { where, } j=1,2 .
$$

The upper surfaces with flexible boundaries are defined by the following fourth order equation

$$
\left(\frac{\partial^{4}}{\partial x^{4}}-\mu^{4}\right) \psi_{j y}-\alpha \psi_{j}=0
$$

where for $j=1$ and $j=2$ the condition is applied at $y=a, x<0$ and $y=b, x>0$, respectively. Here the non-dimensional parameters $\mu$ is the in vacuo plate wavenumber and $\alpha$ a fluid loading parameter defined by

$$
\mu^{4}=\frac{12\left(1-v^{2}\right) c^{2} \rho_{p}}{k^{2} h^{2} E}, \quad \alpha=\frac{12\left(1-v^{2}\right) c^{2} \rho}{k^{3} h^{3} E},
$$

where $E$ is Young's modulus, $\rho_{p}$ is the density of the plate and $v$ is Poisson's ratio. At $x=0^{-}$, the rigid vertical flanged-strip is given by

$$
\frac{\partial \psi_{1}}{\partial x}=0, \quad 0<y<h_{2}
$$

whereas, at $x=0^{+}$the soft back of strip is defined as

$$
\psi_{2}=0, \quad y \in\left(h_{1}, h_{2}\right) \cup(a, b)
$$

At the aperture $x=0, h_{2}<y<a$, the fluid pressure and the normal component of velocity are continuous, that is

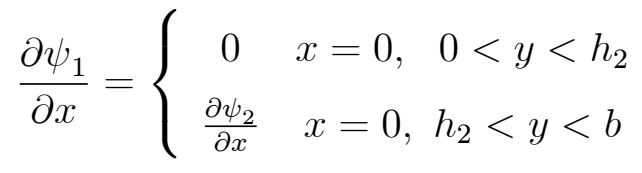

and

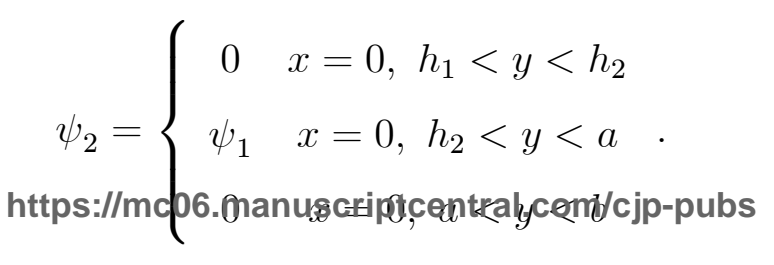


It is well-studied concept that the mathematical model for structures involving flexible boundaries is no more well-posed unless some extra conditions are imposed to make it wellposed. The choice of these conditions depend on the order of flexible boundary, that is, the number of extra conditions is the half of the order of boundary conditions [21]. Moreover these conditions are termed as "edge conditions" which ensure that how the flexible boundaries are connected vertically. In this paper we will discuss the solution for three set of edge conditions; i) clamped edge conditions, ii) pin-jointed edge conditions and iii) pivoted edge conditions.

\section{Acquisition of Solution}

It is well established that there exist many physical situations that can be modelled in terms of waveguides with higher order surfaces. The mathematical models for waveguide structures having plannar boundaries are solvable using the standard Wiener-Hopf technique. In such cases the eigensystem appeared to be Sturm-Liouville (SL) and therefore separation of variables yields the simplest form of eigenfunction expansions that are orthogonal and linearly independent. However, for waveguide structures having geometric discontinuity (abrupt change in height) and comprising of higher order boundaries can be solved using mode-matching approach. In such situations the eigensystem is non Sturm-Liouville, obtained eigenfunctions are linearly dependent that do not satisfy the usual orthogonality relation (OR). In this way the appropriate orthogonality relations are developed by which the Fourier coefficients of eigenfunction expansions can explicitly be expressed in terms of known boundary data. Few extra conditions such as edge conditions are also used to ensure the uniqueness of the mode-matching solution. The choice of these extra conditions certainly affects the scattered field thereby incorporating the physical behavior of scattering process. The detail study of edge condition is referred for instance to [21-24]. With this, the solution to the above stated problem can be achieved while taking the following footsteps; I) to obtain the eigenfunction expansion of duct modes; II) to obtain the related dispersion relations and appropriate orthogonality relations, III) to apply mode-matching at vertical interface and, IV) the implication of appropriate edge conditions. 


\subsection{Eigenfunction Expansion}

Consider an incident wave of harmonic time dependence propagating from negative $x$-axis towards $x=0$. The incident wave is considered with an arbitrary duct mode. At $x=0$ it will scatter into an infinite number of reflected and transmitted modes. With the help of separation of variables, the eigen expansion of these scattered duct modes are given by

$$
\psi_{1}(x, y)=F_{\ell} \cosh \left(\tau_{\ell} y\right) e^{i \eta_{\ell} x}+\sum_{n=0}^{\infty} A_{n} \cosh \left(\tau_{n} y\right) e^{-i \eta_{n} x}
$$

and

$$
\psi_{2}(x, y)=\sum_{n=0}^{\infty} B_{n} \cosh \left[\gamma_{n}\left(y-h_{1}\right)\right] e^{i s_{n} x},
$$

where the first term in above (9) denotes the incident field. The forcing $F_{\ell}=\sqrt{\alpha / C_{\ell} s_{\ell}}$ (where the quantity $C_{\ell}$ will be defined later) is chosen for algebraic convenience and to scale the incident power at unity as well. The counter $\ell$ is considered to incorporate two different incident duct modes, that is, $l=0$ for fundamental mode incident whereas $l=1$ for the secondary mode incident. The quantities $\eta_{n}=\sqrt{\tau_{n}^{2}+1}$ and $s_{n}=\sqrt{1+\gamma_{n}^{2}}$ are the wave numbers of $n^{\text {th }}$ reflected and transmitted modes, respectively. These wave numbers are may have real or imaginary parts depending upon the values of $\tau_{n}$ and $\gamma_{n}$.

\subsection{Dispersion and Orthogonality Relations}

It can be seen that condition (3) contains even order derivative which ensures the even order of corresponding characteristic polynomial [20]. Therefore the eigen values $\tau_{n}$ and $\gamma_{n} ;(n=0,1,2 \ldots)$ of the duct eigenfunction satisfy the following complicated dispersion relations

$$
\left(\left(\tau_{n}^{2}+1\right)^{2}-\mu^{4}\right) \tau_{n} \sinh \left(\tau_{n} b\right)-\alpha \cosh \left(\tau_{n} b\right)=0
$$

and

$$
\left(\left(\gamma_{n}^{2}+1\right)^{2}-\mu^{4}\right) \gamma_{n} \sinh \left[\gamma_{n}\left(b-h_{1}\right)\right]-\alpha \cosh \left[\gamma_{n}\left(b-h_{1}\right)\right]=0
$$

The equations (11)-(12) can be solved numerically for $\tau_{n}, \gamma_{n}$ which in turn, satisfy the properties mentioned and proved in many articles, for example [9, 15, 25].

For the problems in which waveguide comprises of walls with soft, hard or impedance type, the separation of variable renders the solution in a simple way. Also the corresponding eigenfunctions satisfy the ordinaryorthogonality relation,Albeit the governing system is 
Sturm-Liouville (SL) in nature. But system will be no more SL if waveguide comprises of flexible boundaries. In such circumstances the eigenfunctions do not satisfy standard orthogonality relations. Thus, for such systems we have to explore the related orthogonal properties. The problem considered herein contain elastic plate bounded duct for $x \in(-\infty, 0) \cup(0, \infty)$ in which the eigenfunctions satisfy the generalized form of ORs [21,25] as follows:

$$
\begin{gathered}
\alpha \int_{0}^{a} \cosh \left(\tau_{m} y\right) \cosh \left(\tau_{n} y\right) d y \\
=C_{m} \delta_{m n}-\left(\tau_{n}^{2}+\tau_{m}^{2}+2\right) \tau_{m} \sinh \left(\tau_{m} a\right) \tau_{n} \sinh \left(\tau_{n} a\right),
\end{gathered}
$$

and

$$
\begin{gathered}
\alpha \int_{h_{1}}^{b} \cosh \left[\gamma_{m}\left(y-h_{1}\right)\right] \cosh \left[\gamma_{n}\left(y-h_{1}\right)\right] d y \\
=D_{m} \delta_{m n}-\left(\gamma_{n}^{2}+\gamma_{m}^{2}+2\right) \gamma_{m} \sinh \left[\gamma_{m}\left(b-h_{1}\right)\right] \gamma_{n} \sinh \left[\gamma_{n}\left(b-h_{1}\right)\right]
\end{gathered}
$$

where

$$
C_{n}:=\frac{\alpha a}{2}+\frac{\alpha \cosh \left(\tau_{n} a\right) \sinh \left(\tau_{n} a\right)}{2 \tau_{n}}+2\left(\tau_{n}^{2}+1\right) \tau_{n}^{2} \sinh ^{2}\left(\tau_{n} a\right)
$$

and

$$
D_{n}:=\frac{\alpha\left(b-h_{1}\right)}{2}+\frac{\alpha \cosh \left(\gamma_{n} b\right) \sinh \left(\gamma_{n} b\right)}{2 \gamma_{n}}+2\left(\gamma_{n}^{2}+1\right) \gamma_{n}^{2} \sinh ^{2}\left[\gamma_{n}\left(b-h_{1}\right)\right]
$$

Here $\delta_{m n}$ is the Kronecker's Delta function. It is important to note that the eigenfunctions corresponding to the eigenvalues $\tau_{n}$ or $\gamma_{n} ; n=0,1,2, \cdots$, are linearly dependent but contain well defined orthogonal properties for elastic plate bounded ducts. Having obtained well defined orthogonal properties the scattered modes coefficients, $\left(A_{n} B_{n}\right) ; n=0,1,2 \ldots$, can be found by using the continuity conditions of pressure and normal velocity at matching interface along with appropriate edge conditions. This process is illustrated in the subsequent section.

\subsection{Mode-Matching}

Mode-matching technique is considered to be the most suitable way of finding the solution for acoustic structural problems. As mentioned earlier that the underlying system is non-SL which on using the mode-matching reduces to an infinite system of algebraic equations which is not well-behaved. The same technique has been utilized by many authors $[9,10,15,26,27]$ in number of disciplines to address the complicated discontinuous geometric structures. Therefore we opt mode-matching across the interface to find the solution of https://mc06.manuscriptcentral.com/cjp-pubs 
above boundary value problem. On using (9)-(10) into (7), it is straight forward to obtain

$$
\begin{gathered}
F_{\ell} \eta_{\ell} \cosh \left(\tau_{\ell} y\right)-\sum_{n=0}^{\infty} A_{n} \eta_{n} \cosh \left(\tau_{n} y\right) \\
=\left\{\begin{array}{cc}
0 & 0<y<h_{2} \\
\sum_{n=0}^{\infty} B_{n} s_{n} \cosh \left[\gamma_{n}\left(y-h_{1}\right)\right] & h_{2}<y<b
\end{array} .\right.
\end{gathered}
$$

On multiplying with $\alpha \cosh \left(\tau_{m} y\right)$, integrating from 0 to $a$ and then using orthogonality relation (13), we get

$$
A_{m}=F_{\ell} \delta_{m \ell}-\frac{\tau_{m} \sinh \left(\tau_{m} a\right)}{\eta_{m} C_{m}}\left\{e_{1}+\left(\tau_{m}^{2}+2\right) e_{2}\right\}-\frac{\alpha}{\eta_{m} C_{m}} \sum_{n=0}^{\infty} B_{n} s_{n} R_{m n},
$$

where

$$
\begin{gathered}
e_{1}=-i \psi_{1 x y y y}(0, a) \\
e_{2}=-i \psi_{1 x y}(0, a)
\end{gathered}
$$

and

$$
R_{m n}=\int_{h_{2}}^{a} \cosh \left(\tau_{m} y\right) \cosh \left[\gamma_{n}\left(b-h_{1}\right)\right] d y
$$

Similarly the continuity of pressure (8) along with appropriate orthogonality relation (14) reveals

$$
B_{m}=\frac{\gamma_{m} \sinh \left[\gamma_{m}\left(b-h_{1}\right)\right]}{D_{m}}\left\{e_{3}+\left(\gamma_{m}^{2}+2\right) e_{4}\right\}+\frac{\alpha}{D_{m}}\left\{F_{\ell} R_{\ell m}+\sum_{n=0}^{\infty} b_{n} R_{n m}\right\}
$$

where

$$
e_{3}=\psi_{1 y y y}(0, b)
$$

and

$$
e_{4}=\psi_{1 y}(0, b)
$$

Here $e_{1}-e_{4}$ are arbitrary constants that can be determined by using the edge conditions. Three sets are edge condition are considered here which we discuss in following cases.

\subsection{Use of Edge Conditions}

As in case of higher order field equation and boundary conditions, the ORs are likely to be non simple then there is inevitably a question of how to impose the corner conditions at the junction of discontinuity. Thus, the development of appropriate OR is not sufficient without a practical and convenient approach of imposing the edge conditions. In order to incorporate

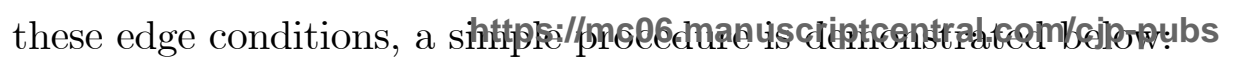




\subsubsection{Clamped edges (zero displacement and zero slope)}

For edges to be clamped, the elastic plate displacement and gradient are assumed as zero. That is,

$$
\begin{aligned}
\frac{\partial \psi_{1}}{\partial y}=0, & (x, y)=(0, a) \\
\frac{\partial^{2} \psi_{1}}{\partial x \partial y}=0, & (x, y)=(0, a) \\
\frac{\partial \psi_{2}}{\partial y}=0, & (x, y)=(0, b)
\end{aligned}
$$

and

$$
\frac{\partial^{2} \psi_{2}}{\partial x \partial y}=0, \quad(x, y)=(0, b)
$$

From (26)-(27), it is straightforward to write $e_{2}=e_{4}=0$. However, to find $e_{3}$ we multiply equation (18) by $\tau_{m} \sinh \left(\tau_{m} b\right)$, taking sum over $m$ upto $\infty$ and then use of edge condition (25) yields

$$
e_{1}=\frac{2 F_{\ell} \tau_{\ell} \sinh \left(\tau_{\ell} a\right)}{S_{1}}-\frac{\alpha}{S_{1}} \sum_{m=0}^{\infty} \sum_{n=0}^{\infty} \frac{B_{n} s_{n} \tau_{m} \sinh \left(\tau_{m} b\right) R_{m n}}{\eta_{m} C_{m}}
$$

where

$$
S_{1}=\sum_{m=0}^{\infty} \frac{\left[\tau_{m} \sinh \left(\tau_{m} a\right)\right]^{2}}{\eta_{m} C_{m}}
$$

Similarly from (22) and (28), it is found that

$$
e_{3}=-\frac{\alpha}{S_{2}} \sum_{m=0}^{\infty} \frac{s_{m} \gamma_{m} \sinh \left[\gamma_{m}\left(b-h_{1}\right)\right]}{D_{m}}\left\{F_{\ell} R_{\ell m}+\sum_{n=0}^{\infty} B_{n} R_{n m}\right\}
$$

where

$$
S_{2}=\sum_{m=0}^{\infty} \frac{s_{m} \gamma_{m}^{2} \sinh ^{2}\left[\gamma_{m}\left(b-h_{1}\right)\right]}{D_{m}}
$$

\subsubsection{Pin-jointed edges}

In this condition we assume the zero displacement and the zero bending moment at the elastic plate edges. That is

$$
\begin{gathered}
\frac{\partial \psi_{1}}{\partial y}=0, \quad(x, y)=(0, a) \\
\frac{\partial^{3} \psi_{1}}{\partial x^{2} \partial y}=0, \quad(x, y)=(0, a) \\
\frac{\partial \psi_{2}}{\partial y}=0, \quad(x, y)=(0, b)
\end{gathered}
$$


and

$$
\frac{\partial^{3} \psi_{2}}{\partial x^{2} \partial y}=0, \quad(x, y)=(0, b)
$$

Now on using the reflected mode coefficient (18) into (9), differentiating with respect to $y$, and then using the edge condition (33), we obtain

$$
e_{1} S_{1}+e_{2} S_{3}=2 F_{\ell} \tau_{\ell} \sinh \left(\tau_{\ell} a\right)-\alpha \sum_{m=0}^{\infty} \sum_{n=0}^{\infty} \frac{B_{n} s_{n} \tau_{m} \sinh \left(\tau_{m} b\right) R_{m n}}{\eta_{m} C_{m}}
$$

where

$$
S_{3}=\sum_{m=0}^{\infty} \frac{\left(\tau_{m}^{2}+2\right)\left[\tau_{m} \sinh \left(\tau_{m} a\right)\right]^{2}}{\eta_{m} C_{m}} .
$$

Similarly with the aid of edge condition (34), we found

$$
e_{1} S_{4}+e_{2} S_{5}=2 F_{\ell} \eta_{\ell}^{2} \tau_{\ell} \sinh \left(\tau_{\ell} a\right)-\alpha \sum_{m=0}^{\infty} \sum_{n=0}^{\infty} \frac{B_{n} s_{n} \eta_{m} \tau_{m} \sinh \left(\tau_{m} b\right) R_{m n}}{C_{m}}
$$

where

$$
S_{4}=\sum_{m=0}^{\infty} \frac{\eta_{m}\left[\tau_{m} \sinh \left(\tau_{m} a\right)\right]^{2}}{C_{m}}
$$

and

$$
S_{5}=\sum_{m=0}^{\infty} \frac{\eta_{m}\left(\tau_{m}^{2}+2\right)\left[\tau_{m} \sinh \left(\tau_{m} a\right)\right]^{2}}{C_{m}} .
$$

Thus, it clearly follows that the values of $e_{1}-e_{2}$ and $e_{4}$ are found to be zero whereas the value of $e_{3}$ can be found by imposing the edge condition (36) together with the Green's function, which follows

$$
\begin{gathered}
e_{3}=\alpha F_{\ell} \sum_{m=0}^{\infty} \frac{s_{m}^{2}\left\{P_{\ell m}+T_{\ell m}\right\} \gamma_{m} \sinh \left[\gamma_{m}\left(b-h_{1}\right)\right]}{D_{m}} \\
+\alpha \sum_{n=0}^{\infty} \sum_{m=0}^{\infty} \frac{B_{n} s_{m}^{2}\left\{P_{n m}+T_{n m}\right\} \gamma_{m} \sinh \left[\gamma_{m}\left(b-h_{1}\right)\right]}{D_{m}}
\end{gathered}
$$

where

$$
P_{m n}=\int_{h_{1}}^{h_{2}} \cosh \left(\tau_{m} y\right) \cosh \left[\gamma_{n}\left(b-h_{1}\right)\right] d y
$$

and

$$
T_{m n}=\int_{a}^{b} \cosh \left(\tau_{m} y\right) \cosh \left[\gamma_{n}\left(b-h_{1}\right)\right] d y
$$




\subsubsection{Pivoted edges}

The pivoted edge conditions are usually feasible only when $a=b$. These can be defined as

$$
\begin{gathered}
\frac{\partial \psi_{j}}{\partial y}=0, \quad(x, y)=(0, b) \quad j=1,2 \\
\frac{\partial^{2} \psi_{1}}{\partial x \partial y}(0, b)=\frac{\partial^{2} \psi_{2}}{\partial y \partial x}(0, b)
\end{gathered}
$$

and

$$
\frac{\partial^{3} \psi_{1}}{\partial x^{2} \partial y}(0, b)=\frac{\partial^{3} \psi_{2}}{\partial y \partial x^{2}}(0, b)
$$

The above conditions can be used to calculate the values of constants $e_{1}-e_{4}$. For that we first reconstruct the scattered field potentials (9)-(10) on using (18) and (22). Now by using the edge condition (44), it is straightforward to write $e_{4}=0$ and

$$
e_{1} S_{1}+e_{2} S_{3}=2 F_{\ell} \tau_{\ell} \sinh \left(\tau_{\ell} a\right)-\alpha \sum_{m=0}^{\infty} \sum_{n=0}^{\infty} \frac{B_{n} s_{n} \tau_{m} \sinh \left(\tau_{m} b\right) R_{m n}}{\eta_{m} C_{m}}
$$

However, the simplification of (45) on using the Green's function [20] related identities leads to

$$
\begin{gathered}
e_{2}-e_{3} S_{2}=\alpha \sum_{m=0}^{\infty} \sum_{n=0}^{\infty} \frac{B_{n} s_{n} \tau_{m} \sinh \left(\tau_{m} b\right) Q_{m n}}{C_{m}} \\
+\alpha \sum_{m=0}^{\infty} \frac{s_{m} \gamma_{m} \sinh \left[\gamma_{m}\left(b-h_{1}\right)\right]}{D_{m}}\left\{F_{\ell} R_{\ell m}+\sum_{n=0}^{\infty} B_{n} R_{n m}\right\}
\end{gathered}
$$

where

$$
Q_{m n}=\int_{0}^{h_{2}} \cosh \left(\tau_{m} y\right) \cosh \left[\gamma_{n}\left(b-h_{1}\right)\right] d y
$$

Furthermore, equation (46) can be simplified by constructing the Green's function which may be expressed as

$$
\begin{gathered}
e_{1} S_{4}+S_{5} e_{2}+e_{3}=2 F_{\ell} \eta_{\ell}^{2} \tau_{\ell} \sinh \left(\tau_{\ell} a\right)-\alpha \sum_{m=0}^{\infty} \sum_{n=0}^{\infty} \frac{B_{n} s_{n} \eta_{m} \tau_{m} \sinh \left(\tau_{m} b\right) R_{m n}}{C_{m}} \\
+\alpha \sum_{m=0}^{\infty} \frac{s_{m}^{2} \gamma_{m} \sinh \left[\gamma_{m}\left(b-h_{1}\right)\right]}{D_{m}}\left\{F_{\ell} P_{\ell m}+\sum_{n=0}^{\infty} B_{n} P_{n m}\right\}
\end{gathered}
$$

\section{Results and Discussion}

In this section the obtained solution is truncated first, and then solved numerically for each

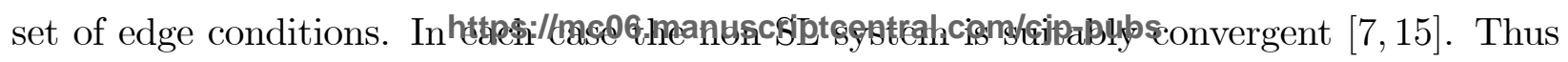


we truncate (18) and (22) together with $e_{1}-e_{4}$ upto $m=0,1, \ldots N-1$ and then solve the retained system simultaneously. The truncated solution can be used to check the accuracy of presented algebra and distribution of energy flux. This not only validate the proposed solution but also provide a useful physical information about the boundary value problem. The reflected power and transmitted power in duct regions [15] are given by

$$
P_{\text {ref }}=\Re e\left\{\frac{1}{\alpha} \sum_{n=0}^{J_{1}}\left|A_{n}\right|^{2} \eta_{n} C_{n}\right\} .
$$

and

$$
P_{\text {trans }}=\Re e\left\{\frac{1}{\alpha} \sum_{n=0}^{J_{2}}\left|B_{n}\right|^{2} s_{n} D_{n}\right\},
$$

respectively, where $J_{1} / J_{2}$ be the cut-on reflected/transmitted modes in two duct regions. Note that the choice of $F_{\ell}=\sqrt{\alpha / C_{\ell} s_{\ell}},(\ell=0$ or 1$)$, certifies that the incident power is unity. Thus

$$
P_{\text {ref }}+P_{\text {trans }}=1
$$

which is conserved power identity. While carrying the numerical computation the elastic plate is chosen of aluminum, of thickness $\bar{h}=0.0006 \mathrm{~m}$ and density $\rho_{p}=2700 \mathrm{~kg} \mathrm{~m}^{-3}$, whereas, values of Young's modulus and Poisson's ratio are taken to be $E=7.2 \times 10^{10} \mathrm{Nm}^{-2}$ and $\nu=0.34$. For each case considered here the speed of sound in air $c=343 \mathrm{~ms}^{-1}$ and density of air $\rho=1.2043 \mathrm{kgm}^{-3}$ are taken from Kaye and Laby [28], whereas, the duct heights are fixed at $\bar{a}=0.06 \mathrm{~m}$ and $\bar{b}=0.085 \mathrm{~m}$.

Now we can discuss the distribution of scattering power against frequency with different set of edge conditions. On taking $\ell=0$ and $\ell=1$ the fundamental (structure-borne) and secondary (fluid-borne) mode, respectively, can be used as an incident field. For the clamped set of edge conditions, reflected power $\left(P_{\text {ref }}\right)$, transmitted power $\left(P_{\text {trans }}\right)$ and their $\operatorname{sum}\left(P_{\text {ref }}+P_{\text {trbns }}\right)$ against frequency $(H z)$ are shown in Figures (2 to 5$)$. These clearly follow that for structure-borne mode incident maximum of the power goes on reflection with and without flanged junction (solid curves in Figures (2\&4)). Whereas, for the fluid-borne mode incident which cuts-on at $f=191 \mathrm{~Hz}$, the transmitted power increases in the absence of flanged junction (dashed curves in Figures (3\&5)). However, the sum of the reflected and transmitted powers (dotted lines) is unity that successfully testifies the conserve power identity (51). For Figures (6-9), the power components are plotted for the pin-jointed set of edge conditions. The powerppropagation behavior for this set of edge conditions is largely 
similar to that of clamped set of edge conditions.

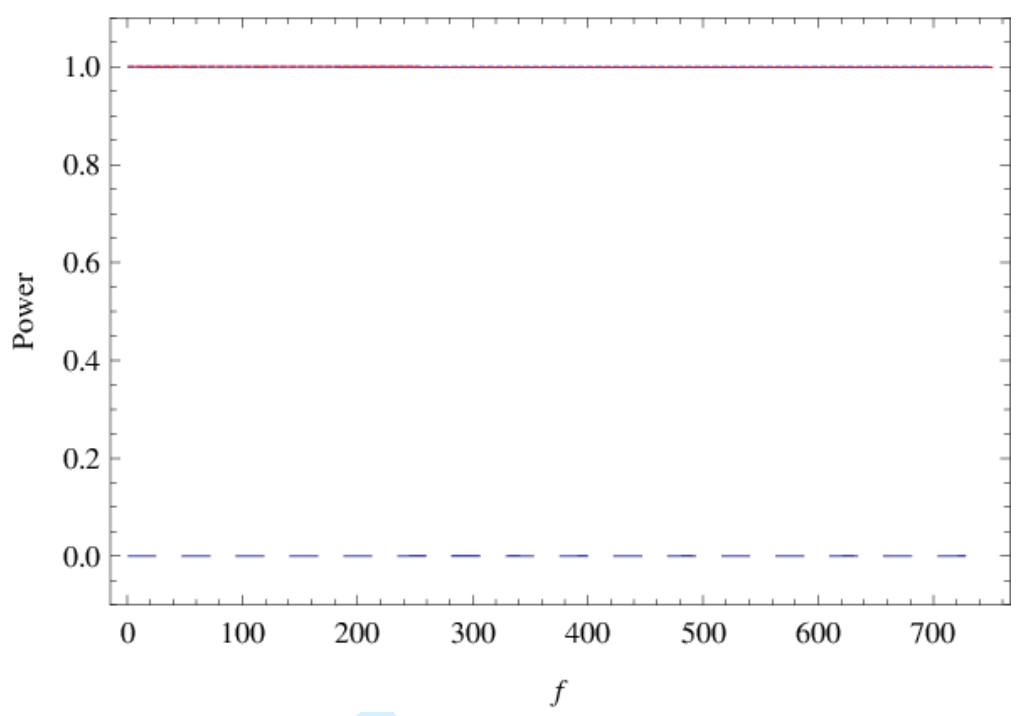

Figure (2): For structure-borne mode incident $(\ell=0)$, the $P_{\text {ref }}$ (solid curve), $P_{\text {trans }}$ (dashed curve) and $P_{\text {ref }}+P_{\text {trans }}($ dotted line) are shown against frequency, where, $\bar{h}_{1}=0.02 m$ and $\bar{h}_{2}=0.04 m$.

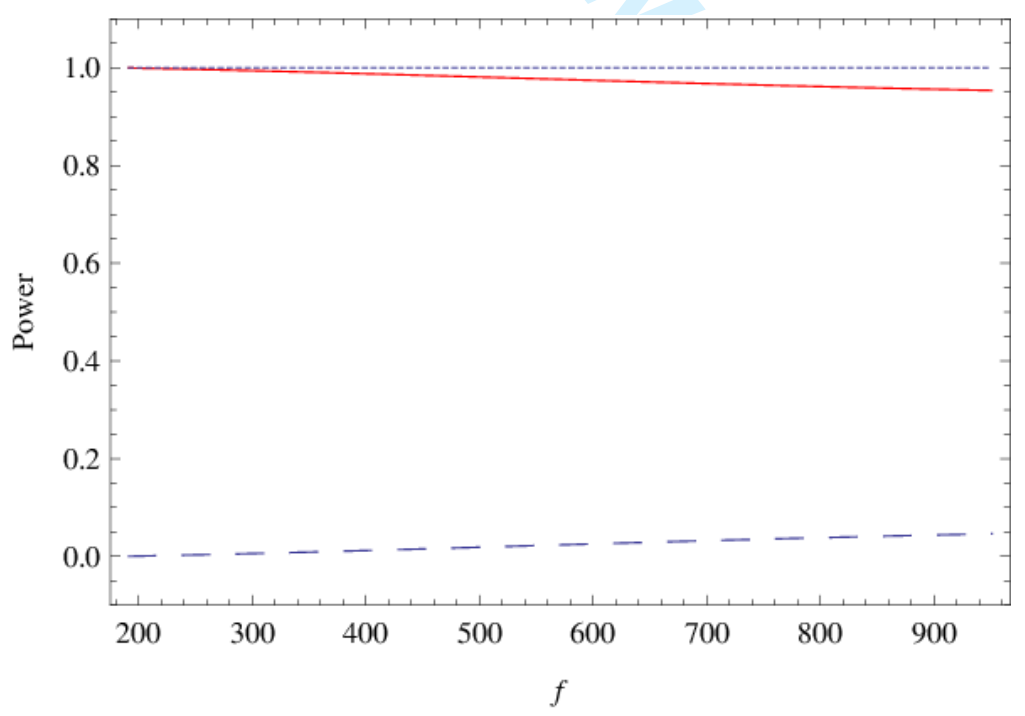

Figure (3): For Fluid-borne mode incident $(\ell=1)$, the $P_{\text {ref }}$ (solid curve), $P_{\text {trans }}$ (dashed curve) and $P_{\text {ref }}+P_{\text {trans }}$ (dotted line) are shown against frequency, where,

$$
\bar{h}_{1}=0.02 m \text { and } \bar{h}_{2}=0.04 m \text {. }
$$




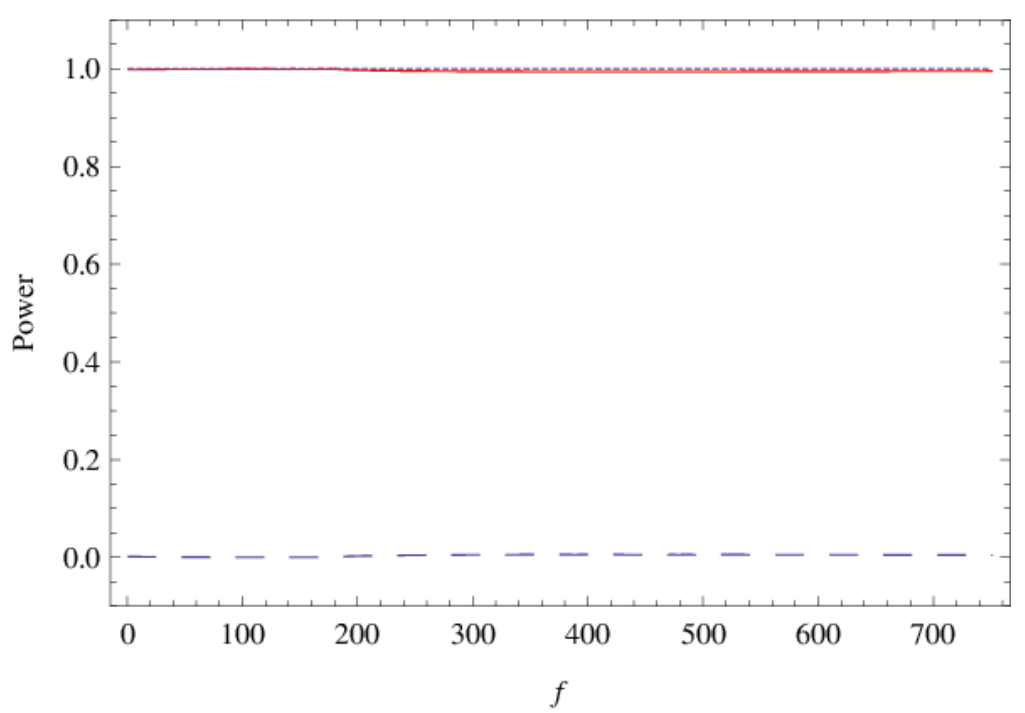

Figure (4): For structure-borne mode incident $(\ell=0)$, the $P_{\text {ref }}$ (solid curve), $P_{\text {trans }}$ (dashed curve) and $P_{\text {ref }}+P_{\text {trans }}($ dotted line) are shown against frequency, where, $\bar{h}_{1}=\bar{h}_{2}=0.02 m$.

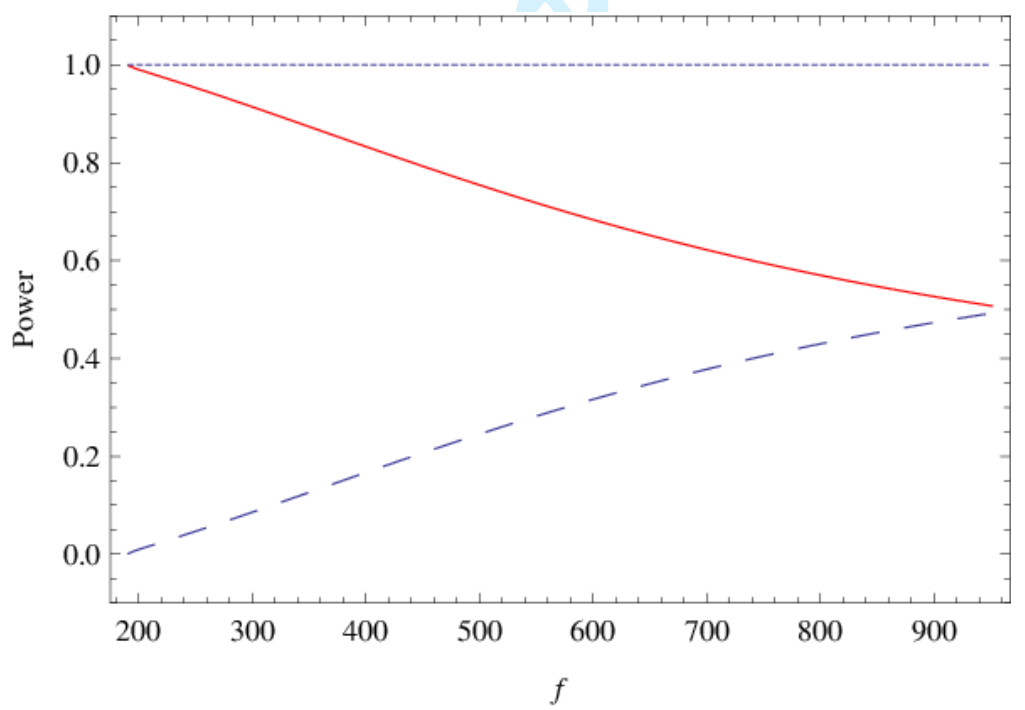

Figure (5): For Fluid-borne mode incident $(\ell=1)$, the $P_{\text {ref }}$ (solid curve), $P_{\text {trans }}$ (dashed curve) and $P_{\text {ref }}+P_{\text {trans }}$ (dotted line) are shown against frequency, where,

$$
\bar{h}_{1}=\bar{h}_{2}=0.02 m
$$

For Figures (6-9) the power components are plotted for the pin-jointed set of edge conditions.

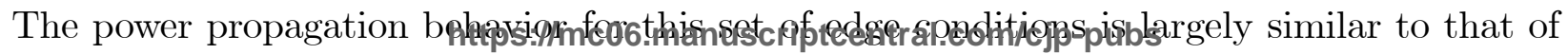


clamped set of edge conditions.

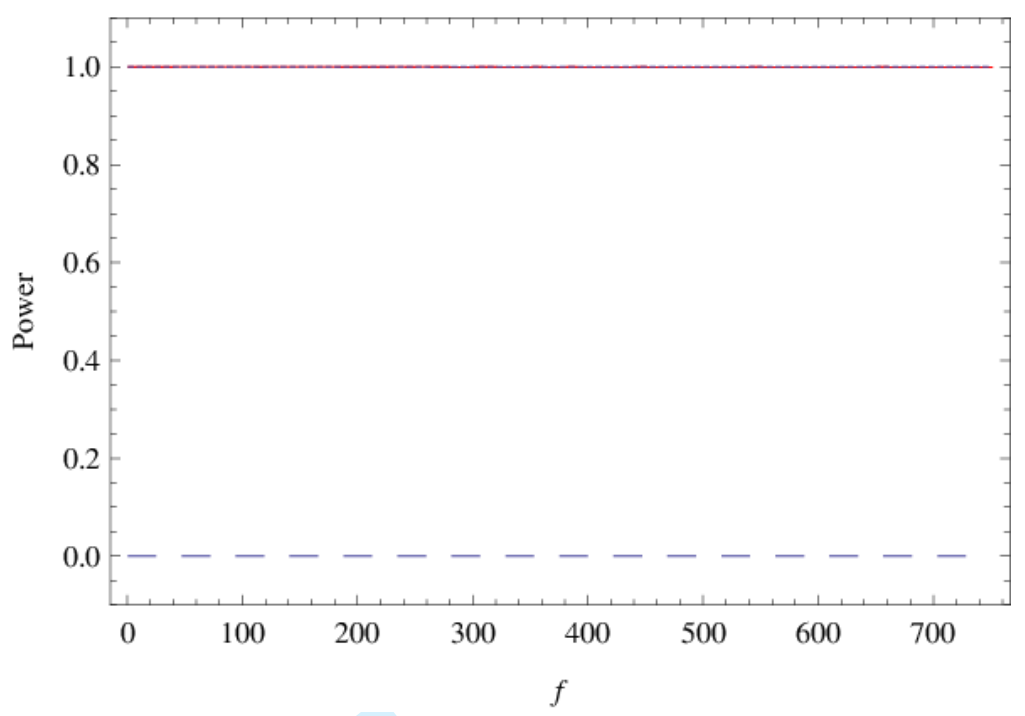

Figure (6): For structure-borne mode incident $(\ell=0)$, the $P_{\text {ref }}$ (solid curve), $P_{\text {trans }}$ (dashed curve) and $P_{\text {ref }}+P_{\text {trans }}($ dotted line) are shown against frequency, where, $\bar{h}_{1}=0.02 m$ and $\bar{h}_{2}=0.04 m$.

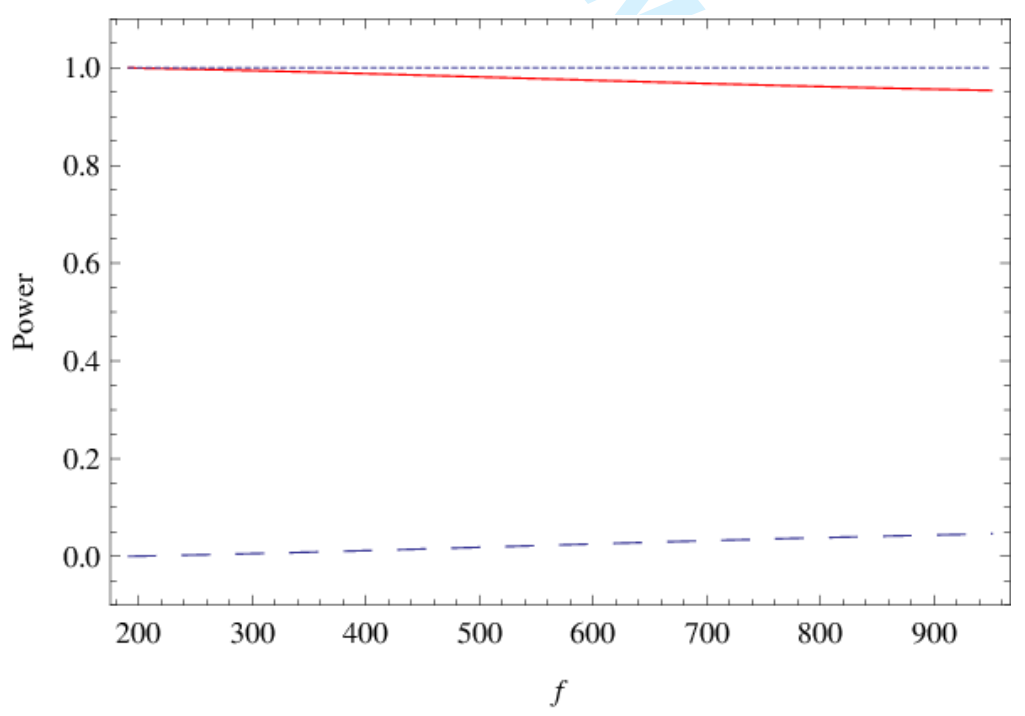

Figure (7): For Fluid-borne mode incident $(\ell=1)$, the $P_{\text {ref }}$ (solid curve), $P_{\text {trans }}$ (dashed curve) and $P_{\text {ref }}+P_{\text {trans }}$ (dotted line) are shown against frequency,, where,

$$
\bar{h}_{1}=0.02 m \text { and } \bar{h}_{2}=0.04 m \text {. }
$$




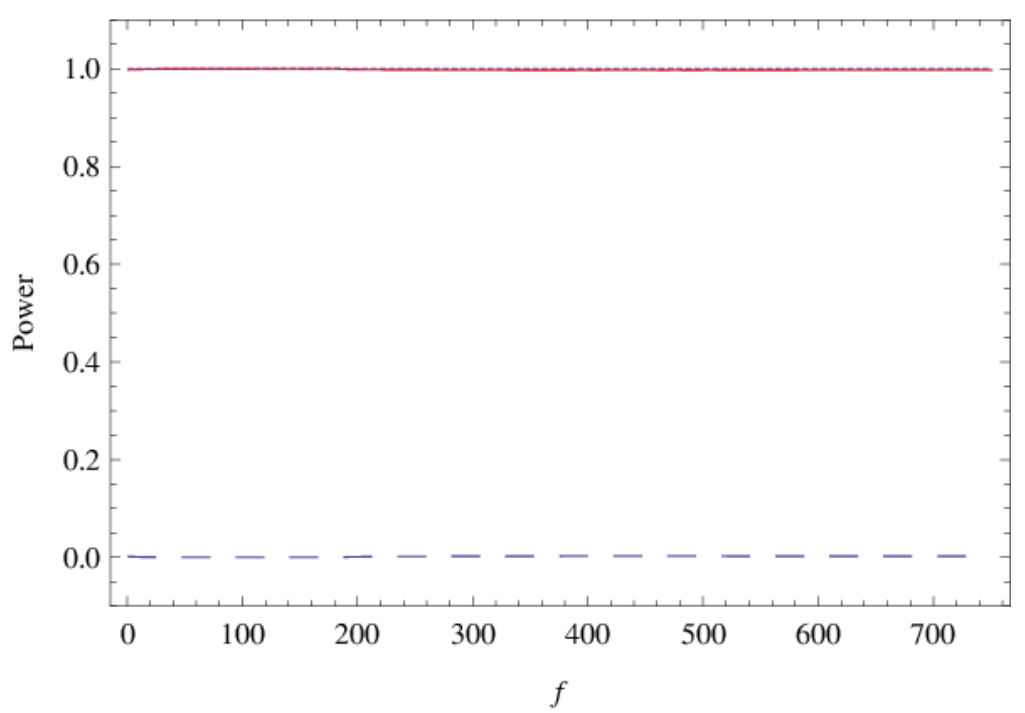

Figure (8): For structure-borne mode incident $(\ell=0)$, the $P_{\text {ref }}$ (solid curve), $P_{\text {trans }}$ (dashed curve) and $P_{\text {ref }}+P_{\text {trans }}($ dotted line) are shown against frequency, where, $\bar{h}_{1}=\bar{h}_{2}=0.02 m$

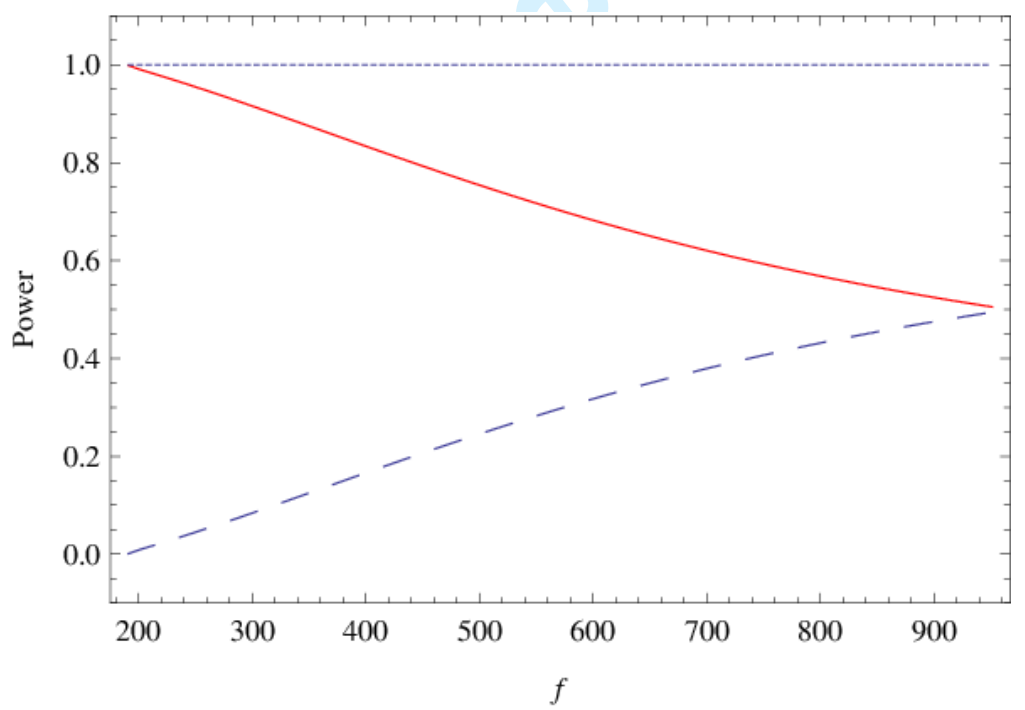

Figure (9): For Fluid-borne mode incident $(\ell=1)$, the $P_{\text {ref }}$ (solid curve), $P_{\text {trans }}$ (dashed curve) and $P_{\text {ref }}+P_{\text {trans }}$ (dotted line) are shown against frequency, where,

$$
\bar{h}_{1}=\bar{h}_{2}=0.02 m
$$

Figures (10-13) depict the power propagation against frequency with pivoted set of edge

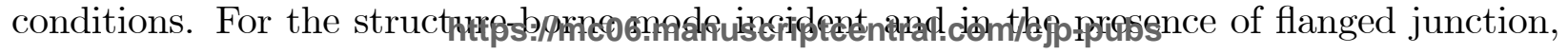


the reflected and transmitted powers tend to have identical power scattering in two duct regions over the variation of frequency (see Fig. 10). However, for the fluid-borne mode incident the reflected power decreases from the maximum to $80 \%$ as frequency varies from $191 \mathrm{~Hz}$ to $953 \mathrm{~Hz}$ (see Fig. 11). Whilst in the absence of flange, the power propagation behavior is quite different (see Figs. 12\&13). As for the case of structure-borne mode incident with $1 \mathrm{~Hz} \leq f \leq 191 \mathrm{~Hz}$, the reflected power decreases steadily upto $70 \%$ of incident power and then goes on to maximum at cut-on frequency $f=191 \mathrm{~Hz}$. But once it crosses the cut-on frequency the reflection decreases upto half of the total power. With this variation of frequency, the transmitted power behaves symmetrically in opposite direction but, of course, the sum of the reflected and transmitted power remains unity (see Fig. 12). On contrary for the fluid-borne mode incident the transmitted power increases from $20 \%$ to $80 \%$ with increasing values of frequency from $191 \mathrm{~Hz}$ to $953 \mathrm{~Hz}$ (see Fig. 13).

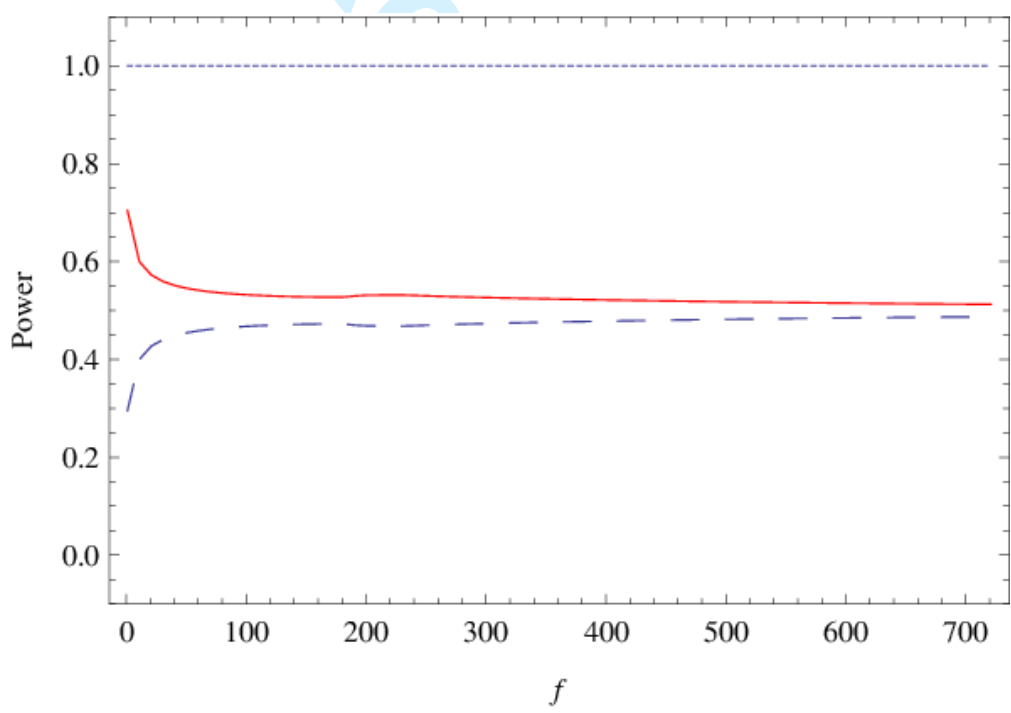

Figure (10): For structure-borne mode incident $(\ell=0)$, the $P_{\text {ref }}$ (solid curve), $P_{\text {trans }}$ (dashed curve) and $P_{\text {ref }}+P_{\text {trans }}$ (dotted line) are shown against frequency, where, $\bar{h}_{1}=0.02 \mathrm{~m}$ and $\bar{h}_{2}=0.04 \mathrm{~m}$. 


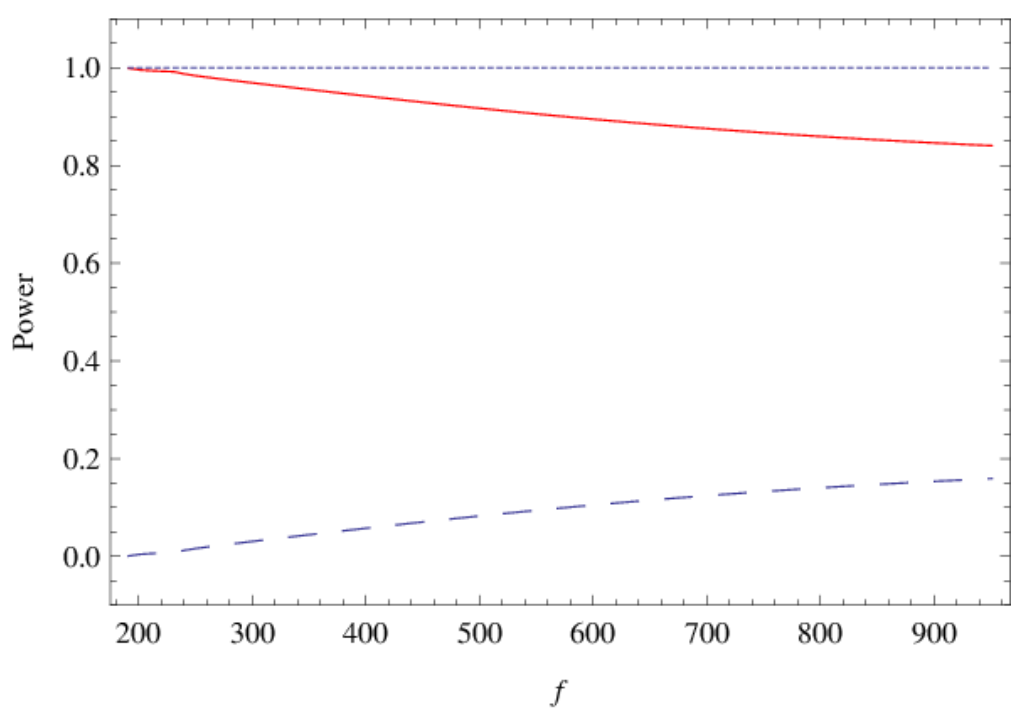

Figure (11): For Fluid-borne mode incident $(\ell=1)$, the $P_{\text {ref }}$ (solid curve), $P_{\text {trans }}$ (dashed curve) and $P_{\text {ref }}+P_{\text {trans }}$ (dotted line) are shown against frequency,, where, $\bar{h}_{1}=0.02 m$ and $\bar{h}_{2}=0.04 m$.

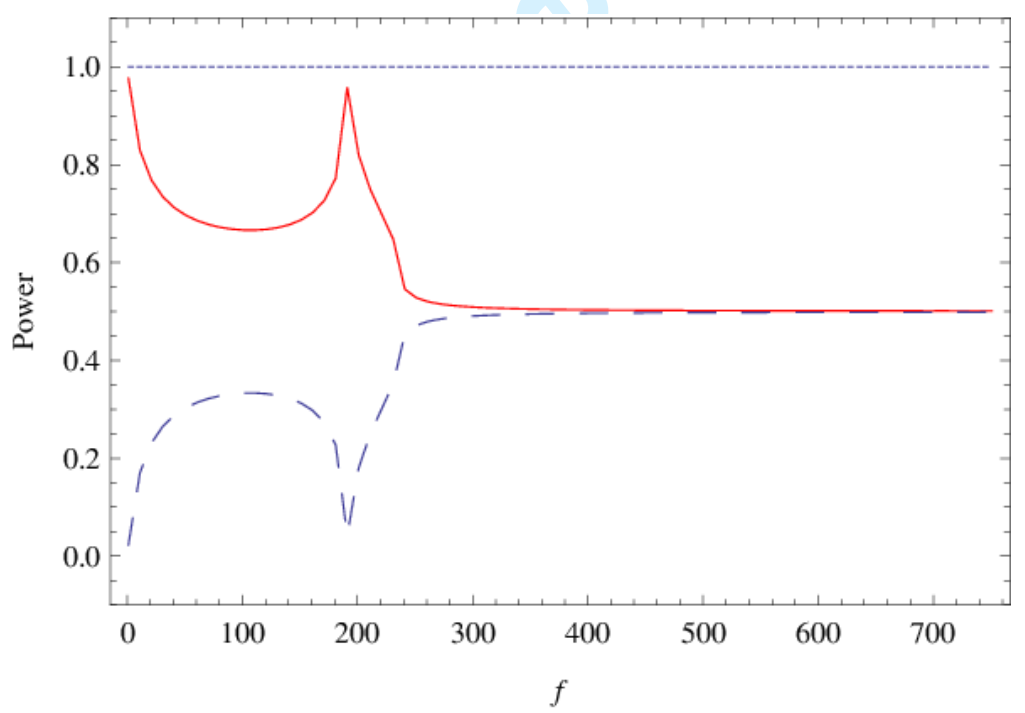

Figure (12): For structure-borne mode incident $(\ell=0)$, the $P_{\text {ref }}$ (solid curve), $P_{\text {trans }}$ (dashed curve) and $P_{\text {ref }}+P_{\text {trans }}($ dotted line) are shown against frequency, where, $\bar{h}_{1}=\bar{h}_{2}=0.02 m$. 


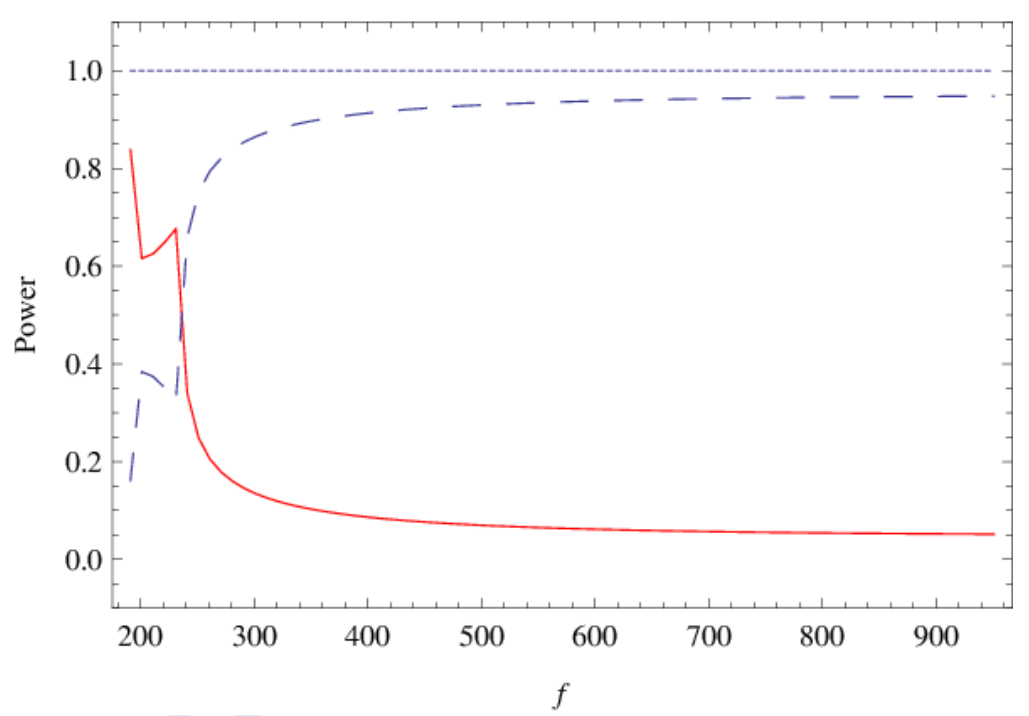

Figure (13): For Fluid-borne mode incident $(\ell=1)$, the $P_{\text {ref }}$ (solid curve), $P_{\text {trans }}$ (dashed curve) and $P_{\text {ref }}+P_{\text {trans }}$

(dotted line) are shown against frequency, where,

$$
\bar{h}_{1}=\bar{h}_{2}=0.02 m \text {. }
$$

Now we reconstruct the continuity conditions at matching interface (7)-(8) by using the truncated solution. Figures (14-17) show the real $(\Re)$ and imaginary ( $\Im)$ parts of nondimensional pressures and normal velocities at matching interface. From these figures it is evident that the pressures and normal velocities match exactly at $x=0, h_{2} \leq y \leq a$. Whereas, $\Re\left\{\psi_{2}(0, y)\right\}$ and $\Im\left\{\psi_{2}(0, y)\right\} \rightarrow 0$ for $y \in\left(h_{1}, h_{2}\right) \cup(a, b)$ (see Figures (14\&15)) and, $\Re\left\{\psi_{1 x}(0, y)\right\}$ and $\Im\left\{\psi_{1 x}(0, y)\right\} \rightarrow 0$ for $y \in\left(0, h_{1}\right)$ (see Figures. (16\&17)). These are exactly the conditions given by equations (7) and (8). Though Figures (16\&17) contain oscillations due to Gibbs phenomenon [29] yet these confirm the accuracy of the modal coefficients. In case this trend increases then we have to cater it by resolving Gibb's phenomenon using the 
Lanczos filter [30-31] thereby confirming the accuracy of velocity conditions more clearly.

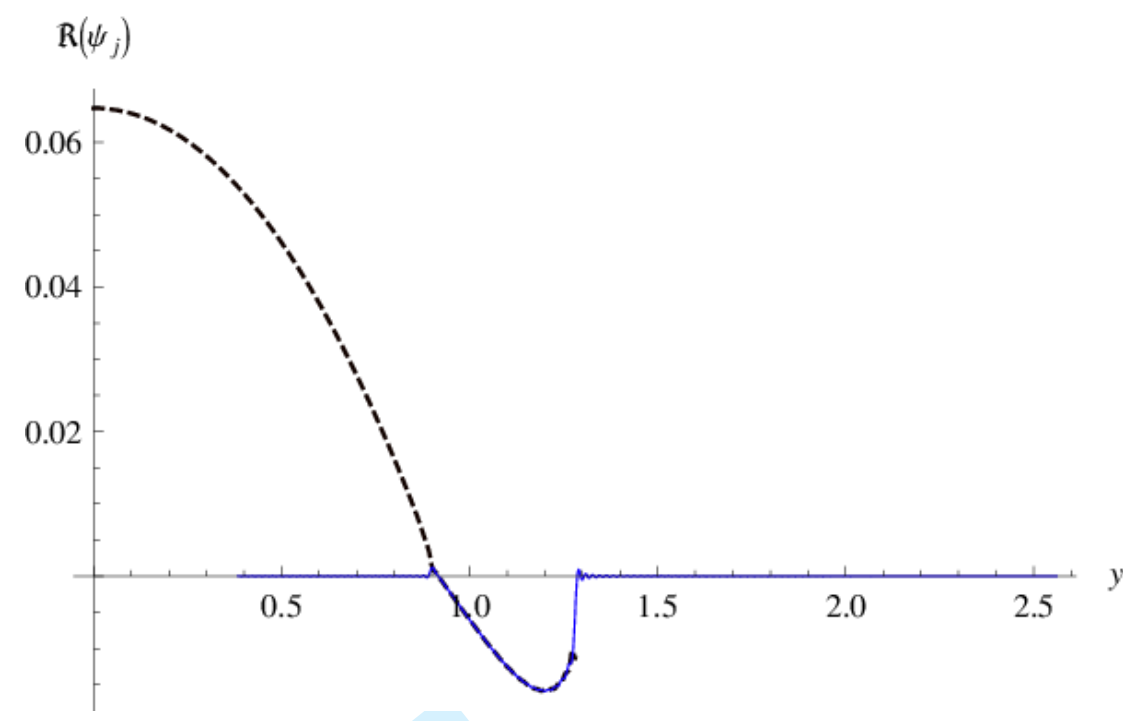

Figure (14): The real part of non-dimensional pressures : $j=1$ (dashed curve, $0 \leq y \leq a$ ) and $j=2$ (solid curve, $\left.h_{1} \leq y \leq b\right)$ are shown for $\bar{a}=0.1 m, \bar{b}=0.2 m, \bar{h}_{1}=0.03 m$ and $\bar{h}_{2}=0.07 m$.

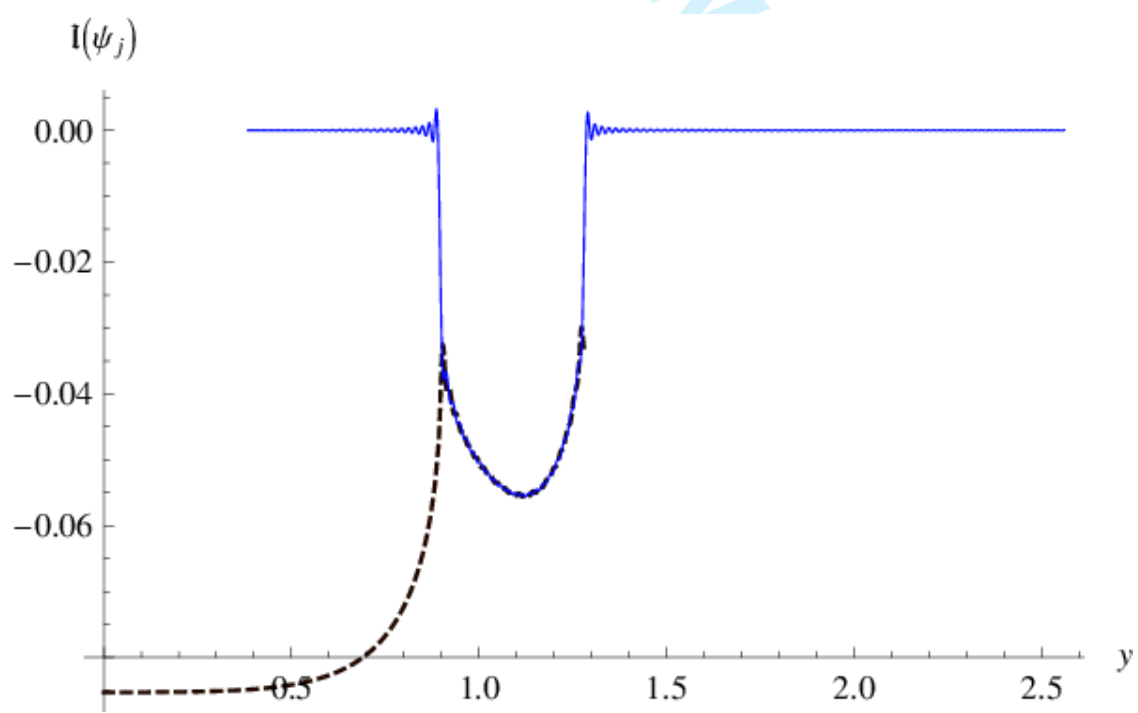

Figure (15): The imaginary part of non-dimensional pressures :

$j=1$ (dashed curve, $0 \leq y \leq a$ ) and $j=2$ (solid curve,

$\left.h_{1} \leq y \leq b\right)$ are shown for $\bar{a}=0.1 m, \bar{b}=0.2 m, \bar{h}_{1}=0.03 m$ and $\bar{h}_{2}=0.07 \mathrm{~m}$. 


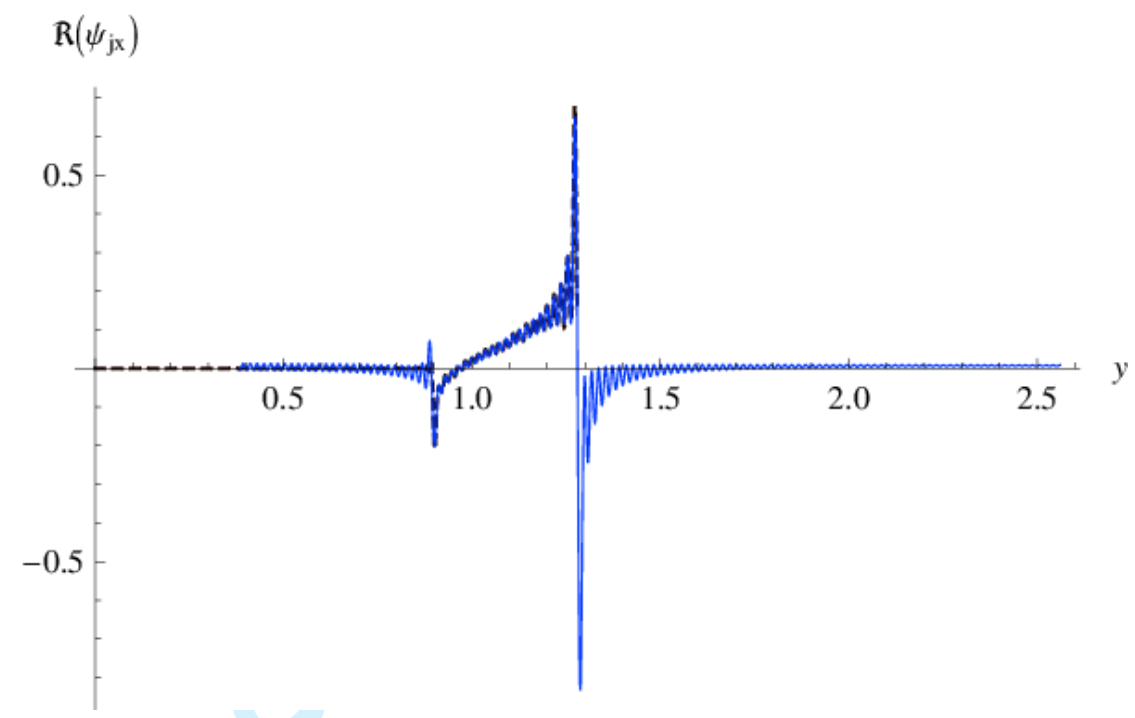

Figure (16): The real part of non-dimensional velocities :

$j=1$ (dashed curve, $0 \leq y \leq a$ ) and $j=2$ (solid curve, $\left.h_{1} \leq y \leq b\right)$ are shown for $\bar{a}=0.1 m, \bar{b}=0.2 m, \bar{h}_{1}=0.03 m$ and $\bar{h}_{2}=0.07 m$.

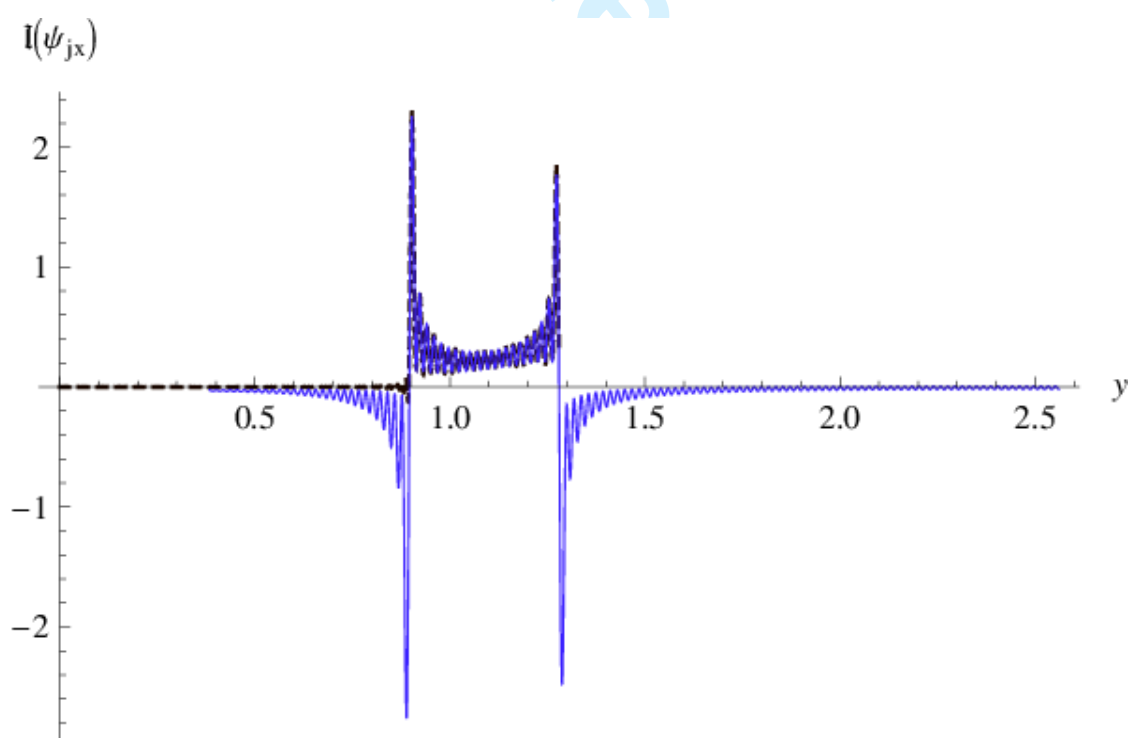

Figure (17): The imaginary part of non-dimensional velocities $: j=1$ (dashed curve , $0 \leq y \leq a$ ) and $j=2$ (solid curve, $\left.h_{1} \leq y \leq b\right)$ are shown for $\bar{a}=0.1 m, \bar{b}=0.2 m, \bar{h}_{1}=0.03 m$ and $\bar{h}_{2}=0.07 m$. 


\section{Conclusion}

The coupled wave scattering characteristics of a two dimensional waveguide structure has been studied using mode-matching approach. The governing problem has been reduced to that of non-SL. The primary focus was the scattering of the incident mode by the flanged junction and, in particular, the effect that different edge conditions have on this process. It is worthwhile to mention that the case of a fully rigid flange is significantly more challenging requiring, for example, a matrix Weiner-Hopf approach. Despite the use of the zero pressure condition, it is concluded that current problem offers much information both about scattering at a flange and about mode-matching approaches. It is observed that the choice of appropriate edge conditions and the incident forcing term expressively affect the scattered field as well as the transmission through structure-borne as compared to fluid-borne vibration. It is also revealed that the power distribution is greatly affected by the attenuated regions and abrupt changes in height of duct. In the end the mode-matching solution is well supported through number of validation points.

Acknowledgments: Authors are thankful to the reviewers for their painstaking review and useful suggestion in improving the quality of paper.

\section{References}

[1] L. Huang, A theoretical study of duct noise control by flexible panels, J. Acoust. Soc. Am. 106, 1801.1809 (1999).

[2] R. Nawaz, M. Ayub, Closed form solution of electromagnetic wave diffraction problem in a homogeneous bi-isotropic medium, Mathematical Methods in the Applied Science, 38 (1), 176-187 (2014).

[3] Mahmood-ul-Hassan, Rawlins, A. D. Sound radiation in a planar trifurcated lined duct, Wave Motion 29, 157-174 (1999).

[4] M. Ayub, R. Nawaz, A. Naeem, Line source diffraction by a slit in a moving fluid, Canadian Journal of Physics, 87 (11) 1139-1149 (2009).

[5] Kaplunov, J., Kirillova, I.V. \& Postnova, Y.A. Dispersion of waves in a plane acoustic

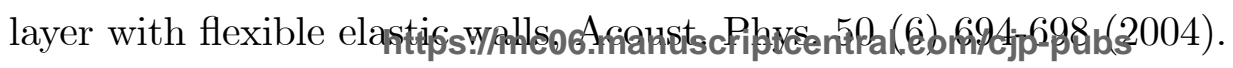


[6] H. Ammari, C. Latiri-Grouz, Electromagnetic scattering on an absorbing plane, Integ. Eqs. and Oper. Theor. 39 159-181 (2001).

[7] Warren, D. P., Lawrie, J. B. and Mohamed, I. M. Acoustic scattering in waveguides with discontinuities in height and material property, Wave Motion, 36, 119-142 (2002).

[8] H. Ammari, G. Bao, Coupling of finite element and boundary element methods for the scattering by periodic chiral structures, J. Comp. Meth. 26(3) 261-283 (2008).

[9] R. Nawaz, M. Afzal, M. Ayub, Acoustic propagation in two-dimensional waveguide for membrane bounded ducts, Comm. Non-Linear Sci. Num. Sim, 20 (2), 421-433 (2015).

[10] JB Lawrie, J. Kaplunov, Edge waves and resonance on elastic structures: an overview, Mathematics and Mechanics of Solids, 17(1), 4-16 (2012).

[11] A. Cummings and I. J. Chang, Sound attenuation of a finite length dissipative flow duct silencer with internal mean flow in the absorbent, J. Sound Vib. 127, 1-17 (1988).

[12] K.S. Peat, A transfer matrix for an absorption silencer element, J. Sound Vib. 146, 353-360 (1991).

[13] R. Kirby, Simplified techniques for predicting the transmission loss of a circular dissipative silencer, J. Sound Vib. 243, 403-426 (2001).

[14] S. N. Panigrahi and M.L. Munjal, Comparison of various methods for analyzing lined circular ducts, J. Sound Vib. 285, 905-923 (2005).

[15] Rab Nawaz and JB Lawrie, Scattering of a fluid-structure coupled wave at a flanged junction between two flexible waveguides, J. Acoust. Soc. Am. 134 (3), 1939-1949 (2013).

[16] K.S. Peat and K.L. Rathi, A finite element analysis of the convected acoustic wave motion in dissipative silencers, J. Sound Vib.184, 529-545 (1995).

[17] R.J. Astley and A. Cummings, A finite element scheme for attenuation in ducts lined with porous material: comparison with experiment, J. Sound Vib. 116, 239-263 (1987).

[18] A.F. Seybert, R.A. Seman and M.D. Lattuca, Boundary element prediction of sound propagation in ducts containing bulk absorbing materials, Transactions of the ASME 120, 976-981 (1998). https://mc06.manuscriptcentral.com/cjp-pubs 
[19] B.Noble, Method based on Wiener-Hopf technique, London:Pergamon Press (1958).

[20] W. Frommhold and F.P. Mechel, Simplified methods to calculate the attenuation of silencers, J. Sound Vib. 141, 103-125 (1990).

[21] JB Lawrie, On eigenfunction expansions associated with wave propagation along ducts with wave-bearing boundaries, IMA Journal of Applied Mathematics 72, 376-394 (2007).

[22] P. R. Brazier-Smith, The acoustic properties of two co-planar half-plane plates, Proceedings of the Royal Society A, 409, 115-139 (1987).

[23] JB Lawrie and ID Abrahams, Scattering of fluid loaded elastic plate waves at the vertex of a wedge of arbitrary angle I: Analytic solution, IMA Journal of Applied Mathematics 59 1-23 (1997).

[24] A. N. Norris, G. R. Wickham, Acoustic diffraction from the junction of two flat plates, Proceedings of the Royal Society A 451, 631-655 (1995).

[25] J. B. Lawrie and I. D. Abrahams, An orthogonality condition for a class of problem with high order boundary conditions: applications in sound/structure interaction, Quarterly Journal of Mechanics and Applied Mathematics 52 (1999), 161-181 (1999).

[26] G. Bao and W. Zhang, An improved mode-matching method for large cavities, IEEE Antennas and Wireless Propagation Letters 4, 393-396 (2005).

[27] G. Bao, J. Gao, J. Lin, and W. Zhang, Mode matching for the electromagnetic scattering from three-dimensional large cavities, IEEE Transactions on Antennas and Propagation, 60, 2004-2010 (2012).

[28] G. W. Kaye and T. H. Laby, Tables of Physical and Chemical Constants (15th Ed.), Longman Scientific \& Technical, UK, (1986).

[29] D. Gottlieb and C.-W. Shu, On the Gibb's phenomenon and its resolution, SIAM Rev. 39(4), 644-668 (1997).

[30] C. E. Duchon, "Lanczos filtering in one and two dimensions," J. Appl. Meteorol. 18, 1016-1022 (1979).

https://mc06.manuscriptcentral.com/cjp-pubs 
[31] H. Vandeven, Family of spectral filters for discontinuous problems, J. Sci. Comput. 8, 159-192 (1991). 